\title{
Super-Resolution for Surveillance Video via Adaptive Block-Matching Registration
}

\author{
T. Lu \\ Hubei Province Key Laboratory of Intelligent Robot \\ Wuhan Institute of Technology \\ Wuhan, China \\ W. Yang \\ Hubei Province Key Laboratory of Intelligent Robot \\ Wuhan Institute of Technology \\ Wuhan, China \\ Y.J. Wan \\ Hubei Province Key Laboratory of Intelligent Robot \\ Wuhan Institute of Technology \\ Wuhan, China
}

\begin{abstract}
In recent years, numerous surveillance cameras are widely installed in the streets, residential areas and traffic routes to protect the national public security. Since interested objectives are often far away from the camera, the resolution of these target images are always in low-resolution (LR). It is hard to recognize the LR monitoring targets. Super-resolution (SR) algorithm can improve the resolution of input images effectively. It takes advantages of multi-frame images to reconstruct high-resolution (HR) target image. However, registration errors reduce the quality of reconstructed HR images. In order to solve this problem, an adaptive block-matching registration method based video SR algorithm is proposed in this paper. According to video content adaptive registration parameters improve the accuracy of multi-frame registration and furthermore enhance the quality of reconstructed HR image. Experimental results show that the proposed SR algorithm has better performance both on subjective and objective reconstruction quality than many state-of-art SR algorithms.
\end{abstract}

Keywords-video surveillance; block-matching; adaptive registration; super-resolution

\section{INTRODUCTION}

Currently, digital video has been widely used in our live. In many practical applications, HR videos or images

are needed to reflect the details. But due to the changes in the surrounding environment, such as the lights, image target motion blur and other factors, most of the surveillance video images resolution is in $\mathrm{LR}$, which affects the recognition about monitoring target. SR technology can take advantage of LR input images to reconstruct HR images, and meet the requirements of application in public security, remote sensing, astronomy and other fields. Since 1964, Harris et al proposed a theoretical basis for SR mathematics to reconstruct HR images, and created a theoretical basis for the SR technology. In 1984, Huang and Tasi [1] proposed the concept of SR image. But their algorithm is based on global translational motion. In order to reflect the general movement of the video image, airspace multi-frame image SR methods were proposed and developed rapidly. Currently the methods of projections onto the convex set (POCS) algorithm [2] and the maximum a posteriori (MAP) algorithm [3] are used widely.

The applications of Video SR technology can be divided into two categories, one is to reconstruct a HR images by Multi-frame LR images, and the other is to obtain a HR video sequence by a LR video sequence. On this basis, many studies [4-6] proposed their algorithms and obtained good reconstruction results. In the current algorithm, the estimation of image motion is one of the key technical indicators, so SR algorithms based on low quality surveillance video images is more challenging. Especially in a complex local motion of video surveillance, adaptive accurate registration method has become an important task.

\section{IMAGE REGISTRATION BY ADAPTIVE BLOCK-MATCHING}

The precision of sub-pixel image registration is an important factor to affect the SR reconstruction. Common registered methods have optical flow algorithm, image feature matching algorithm and Block-Matching image registration algorithm [7]. Optical flow algorithm has the high accuracy of matching, but it would be suffered by noise easily. Image feature matching algorithm is difficult in extracting features in the complex monitoring conditions. So the adaptive Block-Matching algorithm is selected. Step 1: image is divided into a series of blocks. Step 2: the minimum error square is found under standard conditions. Step 3: the displacement vector is obtained. As shown in Fig.1.

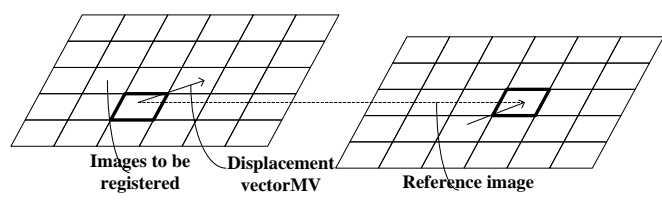

FIGURE I. DIAGRAM OF BLOCK-MATCHING

Sum of Absolute Difference (SAD) is used as matching criteria. SAD is shown as follows:

$$
S A D(i, j)=\frac{1}{M N} \sum_{x=1}^{M} \sum_{y=1}^{N}|f(x, y)-g(x+i, y+j)|
$$

The $(i, j)$ is the displacement vector, $f(x, y)$ is the gray value of image to be registered, $g(x, y)$ is the gray value of reference image, $M \times N$ for the selected block size,

In order to get a balance of estimation accuracy and computation accuracy, suitable match block size is needed to 
seek. Firstly, edge information between images is found, then establish adaptive guidelines to select block size by the information.

Frame difference method is selected to extract motion information. Step1: the adjacent frames A and B are obtained in the video to subtract pixel-by-point between the corresponding pixel values and get a residual image C. Step2: a threshold value $T$ is set to filter the residual image $C$. The gray value of pixels is less than $T$ set 0 and others set 1 . Step3: the result images are saved as $\mathrm{C} 1$ which can reflect the local movement of objects.

For the image $\mathrm{C} 1$, adaptive criterion is obtained by statistical methods. First, C1 is divided into larger block. Second, for each block, the number of pixels whose gray value is zero is count. Third, a threshold value $S$ is given. If the number exceeds the threshold $S$, the block can be considered that contains a large amount of edge movement information and needs to be reclassified by a smaller block.

Next adaptive threshold correction registration methods are given. Compensated image can be expressed by $g(x 1-m v x(x), x 2-m v y(x))$. For the pixel x, the absolute difference between the registering image and compensated image is DFD, and the absolute difference values between two adjacent frame images is $F D_{x}$

$$
\begin{gathered}
D F D x=\left|f_{x}-g_{[x 1-m v x(x), x 2-m v y(x)]}\right| \\
F D_{x}=\left|f_{x}-g_{x}\right|
\end{gathered}
$$

The image to be registered is $\mathrm{f}$, the reference image is $\mathrm{g}$, $m v x$ and mvy indicates the rows and columns of the motion vector matrix. An image has $\mathrm{N}$ pixels, $x\left(x_{1}, x_{2}\right)$ is the current pixel.

The average value is $\mu_{D F D}$, and variance is $\sigma_{D F D}^{2}$, then:

$$
\begin{gathered}
\mu_{D F D}=\frac{1}{N} \sum_{f} D F D_{x}=\frac{1}{N} \sum_{f}\left|f_{x}-g_{[x 1-m v x(x), x 2-m v y(x)]}\right| \\
\sigma_{D F D}^{2}=\frac{1}{N-1} \sum_{f}\left(D F D_{x}-\mu_{D F D}\right)^{2}
\end{gathered}
$$

A threshold value $T=\mu_{D F D}+2 \sigma_{D F D}$ is set. If registration error exists, DFD should be larger than the threshold value T, and $F D_{x}$ should be a small value.

\section{Video SR Algorithm Based on AdAptive IMAge BLOCK- MATCHING REGISTRATION}

In the previous paragraph, adaptive Block-Matching image registration is researched and analyzed to get accurate adaptive block-Matching registration parameters, then reconstruct a HR images.

The HR physical Scene can be transformed into a sequence of low resolution images. As shown in Fig. 2

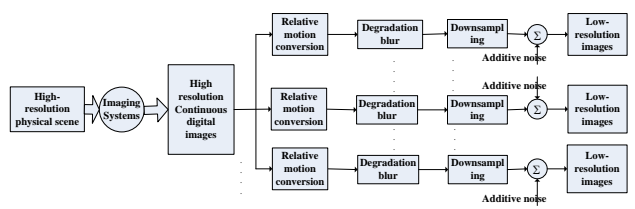

FIGURE II. GENERATION MODEL OF LR VIDEO IMAGES

SR reconstruction is the reverse process of this model. LR images can be transformed into a HR image. The imaging model is shown as follows:

$$
\begin{gathered}
{\left[\begin{array}{l}
Y_{1} \\
\cdot \\
Y_{p}
\end{array}\right]=\left[\begin{array}{ccc}
D_{1} & H_{1} & F_{1} \\
\cdot & \cdot & \cdot \\
\cdot & \cdot & \cdot \\
D_{\mathrm{p}} & H_{\mathrm{p}} & F_{p}
\end{array}\right] X+\left[\begin{array}{l}
E_{1} \\
\cdot \\
\cdot \\
E_{p}
\end{array}\right]} \\
Y_{k}=D_{k} H_{k} F_{k} X+E_{k}
\end{gathered}
$$

where $X$ represents original HR images, $Y$ is a LR image, the frames is $K, F$ is geometric distortion degradation (main represent image translational motion), $H$ is imaging blur degradation, $D$ is image under-sampling degradation, $\mathrm{E}$ is additive noise degradation. IF $Y$ is given, the prior knowledge is used to estimate the value $D F H E$. Then $X$ is obtained by the formulate (7). $C_{k}=D_{k} H_{k} F_{k}$, and $Y_{k}=C_{k} X+E_{k}$ can be written as $Y=C \cdot X+E$.

So single image reconstruction is obtained by cost function:

$$
\arg (X)=\min _{X}\left\lfloor\|Y-C \cdot X\|_{2}^{2}+\lambda \tau(X)\right\rfloor
$$

where $X$ is the high resolution image frames, $C$ is registration coefficient including registration parameters, $\lambda$ is the balance factor to adjust smooth relationship between reconstruction error and reconstruction HR images. $\tau(\mathrm{X})$ is the HR image smooth priori. Equation (8) presents the steepest descent method [8].

Image SR video sequence reconstruction is the reverse process of LR video images, and the SR reconstruction process can be summarized as following 


\begin{tabular}{l} 
Algorithm Video SR based on adaptive block-matching \\
registration \\
\hline Input: frames LR images $Y_{k}$. \\
Output: High resolution image sequence $X_{k}$. \\
(1): Determine a reference frame of the low resolution image \\
frame \\
(2): Use equation (2) to get DFD between the reference frame \\
images, \\
(3): Use equation (5) select adaptive image registration \\
compensation parameter, \\
(4): Calculate the registration parameter matrix by registration \\
compensation parameters and known Down-sampling matrix \\
and fuzzy Matrix. \\
(5): Use Equation (8) to get HR images, \\
(6): Multi-frame HR images combined into HR sequence and \\
output $X_{k}$. \\
\hline
\end{tabular}

\section{Simulation EXPERIMENTS}

Experiment objects: Standard test sequences Foreman and Mobile; Multi-frame LR images as input are obtained by sampling the standard images sequence. Test sequence resolution size is $352 \times 288$ pixels, LR image size is $176 \times 144$ pixels, and magnification is 2 .

Step1: Get the degradation of HR adjacent multi-frame video images. Step2: Test the traditional SR image algorithm based on single partition block size registration, and get its PSNR (Peak Signal to Noise Ratio) and compared to algorithms [6], Step3: Test the SR image algorithm based on adaptive block-matching registration and gets its PSNR. Step4: Contrast these algorithms both by subjective observations and PSNR values.

Using the previous parameter settings, the HR output with proposed method is generated. The resolution of output is $352 \times 288$ pixels. In the same time, many other SR algorithms are used to compare the subjective quality images. As shown in fig3.

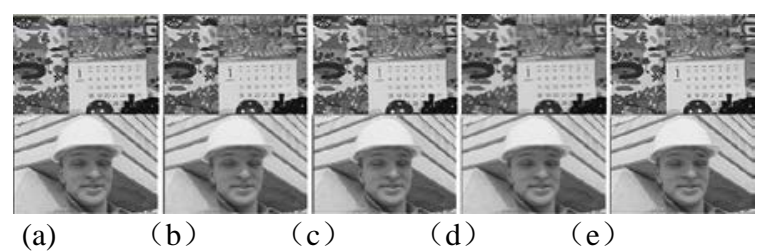

FIGURE III. FIGURE 3: THE SUBJECTIVE COMPARISON CHART OF MULTIPLE SUPER-RESOLUTION RECONSTRUCTION. (A) COLUMN. $(4 \times 4$ BLOCK SIZE), AND NO ERROR CORRECTION OF THE SUPER-RESOLUTION; (B) COLUMN. $(8 \times 8$ BLOCK SIZE), AND NO ERROR CORRECTION OF THE SR; (C) THE RESULTS [6]; (D) CUBIC INTERPOLATION ALGORITHM; (E) RESULTS FROM PROPOSED ALGORITHM.

TABLE I. PSNR OF DifFERENT SR ALgORITHMS.

\begin{tabular}{|c|c|c|c|c|c|}
\hline $\begin{array}{c}\text { Algorith } \\
\text { m }\end{array}$ & \multicolumn{1}{|c|}{ a } & b & \multicolumn{1}{|c|}{ c } & \multicolumn{1}{c|}{ d } & e \\
\hline Mobile & 19.95 & 19.802 & $\begin{array}{l}20.15 \\
2\end{array}$ & $\begin{array}{l}20.03 \\
2\end{array}$ & 20.623 \\
\hline Foreman & 28.82 & 28.74 & $\begin{array}{l}29.15 \\
2\end{array}$ & $\begin{array}{l}29.13 \\
3\end{array}$ & 29.308 \\
\hline
\end{tabular}

From table1, the following conclusions can be drawn: First, SR image algorithm based on single partition block size registration has the better effect on background than up-sample, but in the edge region exists more errors. Second, the adaptive threshold could effectively improve the quality of image registration, and it even gets a smaller PSNR value than cubic up-sample method. Third, SR image algorithm based on adaptive block-matching registration has a better subjective observation than others.

\section{CONCLUSION}

In this paper, a novel video SR algorithm based on adaptive block-matching registration is proposed. During the block-matching registration process, the big block matching has the better anti-noise performance and a simple implement, but it has the poor effect in estimating local movement. At the same time, the smaller block for matching has a good effect in estimating local movement, but it would be easily suffered by noise with more computational complexity. In order to solve this problem, adaptive Block-Matching Algorithm and image registration algorithm are proposed and adaptive threshold parameters can be estimated by the error correction criterion. Finally, these algorithms are applied to the video super-resolution reconstruction algorithm, with video multi-frame block registration techniques to enhance the objective and subjective quality of SR image.

\section{ACKNOWLEDGEMENT}

This work is supported by the National High-Tech Research and Development Program (863Program,No:2013AA12A202), the National Natural Science Foundation of China(61172173), the Natural Science Foundation of Hubei Province of China (2012FFA099,2012FFA134,2013CF125，2014CFA130), the key project and the excellent youth science and technology innovation team project of the Educational Department of Hubei Province of China (No.D20141505,T201206), the scientific research foundation of Wuhan Institute of Technology.

\section{REFERENCES}

[1] Tsai R.Y., Huang, T.S., Multiframe Image Restoration and Registration.In : In Huang, T.S. Advances in Computer Vision and Image Processing, JAI Press, pp: 317-339, 1984.

[2] Patti A.J., Sezan M.I., Tekalp A.M., SR Video Reconstruction With Arbitrary Sampling Lattices and Nonzero Aperture Time. Image Processing, IEEE Transactions on, 6 (8), pp:1064-1076, 1997.

[3] Schultz R.R., Stevenson, R.L., Extraction Of High Resolution Frames From Video Sequences . Image Processing, IEEE Transactions on , 5(6), pp:996-1011,1996.

[4] Baker S., Kanade T., SR Optical Flow[R], Technical Report CMU-RI-TR- 99-36,The Robotics Inst,Carnegie Mellon University,1999.

[5] Bishop, CM Blake, A Marthi, BSR Enhancement of Video. AISTATS, PP:1-8, 2003

[6] Sina Farsiu, Michael Elad, and Peyman Milanfar, Multiframe Demosaicing and Super-Resolution of Color Images. IEEE TRANSACTIONS ON IMAGE PROCESSING, 15(1), pp:141-159, 2006.

[7] Keren, D., Peleg, S., Brada, R., Image Sequence Enhancement Using Sub-pixel Displacements .IEEE Conference on Computer Vision And Pattern Recognition, pp:742-746, 1988

[8] Tikhonov A.N., Regularization of Incorrectly Posed Problems .Soviet Mathematical Doklady, 4, pp:1624-1627, 1963. 